\title{
FRACIONAMENTO DENSIMÉTRICO COM POLITUNGSTATO DE SÓDIO NO ESTUDO DA PROTEÇÃO FÍSICA DA MATÉRIA ORGÂNICA EM SOLOS ${ }^{(1)}$
}

\author{
Paulo Cesar Conceição ${ }^{(2)}$, Madalena Boeni ${ }^{(3)}$,Jeferson Dieckow $^{(4)}$, \\ Cimélio Bayer ${ }^{(5)}$ \& João Mielniczuk ${ }^{(5)}$
}

\begin{abstract}
RESUMO
O fracionamento físico densimétrico é uma técnica que permite estudar a proteção física da matéria orgânica (MO) no solo, sendo utilizadas, de forma indistinta, soluções densas de iodeto de sódio (NaI) e de politungstato de sódio (PTS). O objetivo deste estudo foi avaliar (a) o efeito de soluções de $\mathrm{NaI}\left(1,8 \mathrm{~g} \mathrm{~cm}^{-3}\right)$ e de PTS $\left(1,8,2,0\right.$ e 2,2 $\left.\mathrm{g} \mathrm{cm}^{-3}\right)$ no rendimento de carbono (C) nas frações leve-livre (FLL) e leve-oclusa (FLO) da MO na camada de 0-5 cm de um Argissolo Vermelho [PVd, Eldorado do Sul (RS)] e de um Latossolo Vermelho [LVdf, Dourados (MS)] e (b) a magnitude da proteção física da MO em agregados no acúmulo de $\mathrm{C}$ orgânico nesses solos submetidos, respectivamente, ao sistema plantio direto por 18 anos (PD) e pastagem (Brachiaria decumbens) por nove anos, em comparação à sua condição em preparo convencional (PC). Em ambos os locais, blocos indeformados de solo foram coletados em experimentos de longa duração; os agregados foram separados nos planos de fraqueza e passados em peneira de 9,51 mm; esse material foi submetido ao fracionamento densimétrico da MO do solo. $\mathrm{O}$ uso da solução de PTS $1,8 \mathrm{~g} \mathrm{~cm}^{-3}$ aumentou de 176 a $727 \%$ o rendimento de C na FLO em comparação à solução de $\mathrm{NaI}$ na mesma densidade, mas não teve efeito no rendimento de $\mathrm{C}$ da FLL da MO do solo. O aumento da densidade da solução de PTS de $1,8 \mathrm{~g} \mathrm{~cm}^{-3}$ para $2,0 \mathrm{~g} \mathrm{~cm}^{-3}$ incrementou de 30 a $99 \%$ o rendimento de $\mathrm{C}$ da FLO; contudo, o aumento da densidade para $2,2 \mathrm{~g} \mathrm{~cm}^{-3}$ resultou em aumento pouco expressivo $(\leq 18 \%)$ no rendimento de C na FLO nos diferentes solos e sistemas de manejo. O uso de NaI no fracionamento densimétrico leva à conclusão errônea de que a proteção física não é um mecanismo importante de estabilização da MO no solo. Por outro lado, a utilização da solução de PTS $2,0 \mathrm{~g} \mathrm{~cm}^{-3}$, a qual é recomendada para o fracionamento
\end{abstract}

\footnotetext{
(1) Recebido para publicação em dezembro de 2006 e aprovado em novembro de 2007.

(2) Professor da Universidade Tecnológica Federal do Paraná - UTFPR. Estrada para Boa Esperança, Km 04, Caixa Postal 157, CEP 85660-000 Dois Vizinhos (PR) E-mail: paulocesar@utfpr.edu.br

(3) Estudante do Programa de Pós-Graduação em Ciência do Solo, Universidade Federal do Rio Grande do Sul - UFRGS. Av. Bento Gonçalves 7712, Caixa Postal 15100, CEP 91501-970 Porto Alegre (RS). E-mail: madaboeni@hotmail.com

(4) Professor do Departamento de Solos e Engenharia Agrícola da Universidade Federal do Paraná - UFPR. CEP 80035-050 Curitiba (PR) E-mail: jefersondieckow@ufpr.br

(5) Professor do Departamento de Solos, UFRGS. Pesquisador do CNPq. E-mail: cimelio.bayer@ufrgs.br; mielni@ufrgs.br
} 
densimétrico devido à sua alta eficiência na recuperação do C da FLO, permitiu constatar que a proteção física da MO em agregados de solo foi responsável por 54 e 23 \% do acúmulo de C orgânico no PVd e LVdf, respectivamente, sob plantio direto e pastagem.

Termos de indexação: iodeto de sódio, oclusão, solos tropicais, plantio direto, pastagem.

\title{
SUMMARY: DENSIMETRIC FRACTIONATION WITH SODIUM POLYTUNGSTATE TO INVESTIGATE PHYSICAL PROTECTION OF SOIL ORGANIC MATTER
}

\begin{abstract}
Through the densimetric fractionation it is possible to study the physical protection of soil organic matter (SOM). High density solutions of sodium iodide (NaI) or sodium polytungstate $(S P T)$ have been indistinctively used for this purpose. This study aimed to assess: (a) the capacity of these two solutions at a density of $1.8 \mathrm{~g} \mathrm{~cm}^{-3}$ (NaI-1.8 and SPT1.8) and of SPT at densities of 2.0 (SPT-2.0) and $2.2 \mathrm{~g} \mathrm{~cm}^{-3}$ (SPT-2.2) to increase $C$ recovery in the free light fraction (FLF) and occluded light fraction (OLF) of the organic matter contained in soil aggregates of the $0-5 \mathrm{~cm}$ layer of an Acrisol [PVd, Eldorado do Sul (RS)] and of a Ferralsol [LVdf, Dourados (MS)]; and (b) the magnitude of the physical protection in promoting soil organic matter accumulation in these soils under no-tillage for 18 years and pasture (Brachiaria decumbens) for 9 years, respectively, both in comparison to conventional-tilled soil. Soil blocks were collected and $<9.51 \mathrm{~mm}$ soil aggregates were separated and submitted to density fractionation of soil OM. The SPT-1.8 increased OLF$C$ by 176 to $726 \%$ in comparison to NaI-1.8, but no effect was observed on FLF-C recovery. By using SPT-2.0, the OLF-C recovery increased by 30 to $90 \%$ in comparison with SPT-1.8, but a lower increment ( $\leq 18 \%$ ) was observed when SPT-2.0 was substituted by SPT-2.2. Density fractionation of OM with NaI solution (low OLF and high FLF) may lead to the erroneous conclusion that physical protection is not an important stabilizing mechanism of SOM in soils under no-tillage or pasture. On the other hand, when using SPT-2.0, which was most efficient in recovering $O L F-C$, the physical protection within aggregates was responsible for $54 \%(P V d)$ and $23 \%$ (LVdf) of the total SOM accumulation in soils under no-tillage and pasture, respectively.
\end{abstract}

Index terms: sodium iodide, SOM occlusion, tropical soils, no-till, pasture.

\section{INTRODUÇÃO}

A estabilidade da matéria orgânica (MO) no solo está relacionada à sua recalcitrância molecular, resultante da composição e arranjo estrutural, à da sua proteção física por oclusão em agregados de solo e da estabilidade química devido à sua interação com componentes minerais (Christensen, 1992; Golchin et al., 1994a,b; Chenu \& Plante, 2006). O fracionamento densimétrico, que se baseia na diferença de densidade entre as frações orgânicas livres e os complexos organominerais, permite obter três frações da MO do solo, sendo a quantidade de $\mathrm{C}$ presente em cada fração relacionada com os diferentes mecanismos de estabilização da MO no solo (Hassink et al., 1993; Zech et al., 1997).

A fração leve-livre (FLL) ou interagregados possui composição comparável àquela de materiais vegetais (Golchin et al., 1994b; Freixo et al., 2002a), e o fator determinante da estabilidade desta fração no solo é a recalcitrância intrínseca da molécula (Sollins et al., 1996). A fração leve oclusa (FLO) ou intra-agregados compreende um diversificado conjunto de compostos orgânicos, com grau de decomposição mais avançado em comparação à FLL (Baldock et al., 1992; Golchin et al., 1994b; Christensen, 2000; Freixo et al., 2002a) e cuja estabilidade no solo resulta, além da recalcitrância molecular, da proteção física decorrente da sua oclusão no interior de agregados de solo (Sollins et al., 1996). A fração pesada (FP) constitui-se numa fração orgânica em estádio mais avançado de decomposição, em estreita associação com os minerais do solo. Nesta fração, em adição aos mecanismos de recalcitrância e de oclusão, a estabilidade química atua como mecanismo de estabilização (Christensen, 1992; Sollins et al., 1996).

No fracionamento densimétrico da MO do solo são utilizadas, de forma indistinta, soluções densas de iodeto de sódio- $\mathrm{NaI}$ (1,1 a 1,8 $\left.\mathrm{g} \mathrm{cm}^{-3}\right)$ e de politungstato 
de sódio-PTS (1,6 a 2,4 $\mathrm{g} \mathrm{cm}^{-3}$ ), em relação às soluções orgânicas que apresentam toxidez elevada (Christensen, 1992). Entretanto, estudo sistemático comparando a eficiência desses produtos ainda não foi realizado, havendo apenas uma menção no artigo de Shang \& Tiessen (2001) a uma possível menor eficiência da solução de $\mathrm{NaI}$ na recuperação de frações leves da MO, mas sem a apresentação de resultados comparativos aos da solução de PTS. A menor eficiência do NaI pode ser decorrente da elevada afinidade e formação de complexos altamente estáveis entre o íon I' e a MO (Yu et al., 1996; Radlinger \& Heumann, 2000; Schmett et al., 2006), o que resultaria em aumento de densidade das frações orgânicas e redução da eficiência na separação das frações leves no fracionamento densimétrico da MO do solo. No que se refere à densidade das soluções de $\mathrm{NaI}$ e PTS, tem sido verificado efeito positivo do aumento da densidade da solução no rendimento de $\mathrm{C}$ da FL da $\mathrm{MO}$ do solo (Golchin et al., 1994a; Shang \& Tiessen, 2001; Sohi et al., 2001), sendo esse efeito mais expressivo na recuperação da FLO do que da FLL (Sohi et al., 2001).

Por outro lado, o NaI é o principal produto que vem sendo utilizado por pesquisadores brasileiros em estudos de fracionamento densimétrico da MO do solo (Roscoe et al., 2001; Freixo et al., 2002b; Pinheiro et al., 2004), sendo também amplamente utilizado por grupos de pesquisa internacionais (Gregorich \& Janzen, 1996; Shang \& Tiessen, 2001; Sohi et al., 2001). Os resultados obtidos utilizando-se soluções com densidade de 1,6 a $1,8 \mathrm{~g} \mathrm{~cm}^{-3}$ desse produto indicam quantidades de $\mathrm{C}$ de cinco a até 20 vezes maior na FLL do que na FLO em solos brasileiros (Roscoe et al., 2001; Freixo et al., 2002a,c; Pinheiro et al., 2004). Outro resultado que tem sido obtido usualmente com o uso da solução de $\mathrm{NaI}$ é que, em termos absolutos, o acúmulo de C na FLL em solos em plantio direto tem sido maior do que o acúmulo de C na FLO, quando comparados à condição de referência em preparo convencional (Freixo et al., 2002a,c; Roscoe \& Buurman, 2003).

Entretanto, há aparente falta de coerência entre esses resultados e os mecanismos que atuam na estabilização da MO no solo. No que se refere à quantidade de $\mathrm{C}$ em cada fração, seria coerente pensar que uma fração como a FLO, com dois mecanismos de estabilização (recalcitrância e oclusão), apresente estoque de C maior do que uma FLL que tem apenas a recalcitrância como mecanismo de estabilização da MO do solo. Em relação ao efeito do plantio direto nas diferentes frações físicas da MO, seria coerente também pensar que o aumento do estoque de C na FLO fosse mais expressivo do que na FLL, em decorrência do ambiente mais favorável à formação e à estabilização de agregados (Silva, 1993; Castro-Filho et al., 1998) e, portanto, à oclusão da MO em solos sem revolvimento e com alta adição de resíduos vegetais.

Com base nos mecanismos teóricos de estabilização da MO no solo e sua ocorrência nos respectivos compartimentos de $\mathrm{C}$ obtidos com o fracionamento densimétrico, a hipótese deste estudo é de que o uso da solução de NaI resulte numa subestimação da FLO nos estudos de fracionamento densimétrico da MO do solo e, em conseqüência disso, em subestimação da importância da proteção física na estabilização e no acúmulo de MO em solos tropicais e subtropicais submetidos a sistemas de manejo sem revolvimento do solo. Os objetivos deste estudo foram avaliar (a) a eficiência da solução de PTS com diferentes densidades na recuperação do C das FLL e FLO em dois solos brasileiros com textura e mineralogia distintas, tomando como referência a solução de $\mathrm{NaI} 1,8 \mathrm{~g} \mathrm{~cm}^{-3}$, e (b) a magnitude da proteção física no acúmulo de MO em solo tropical sob pastagem e em solo subtropical sob plantio direto.

\section{MATERIAL E MÉTODOS}

O estudo foi baseado em amostras de solo de dois experimentos de longa duração, sendo um localizado sob clima subtropical, em Eldorado do Sul (RS), e outro sob clima tropical, em Dourados (MS). O primeiro experimento, instalado em 1985, está localizado na Estação Experimental Agronômica da UFRGS, sobre um Argissolo Vermelho distrófico-PVd (Embrapa, 1999) textura franco-argilo-arenosa. O clima da região, segundo classificação de Köppen, é subtropical de verão úmido quente, do tipo fundamental $\mathrm{Cfa}$, e a precipitação pluvial média anual é de $1.440 \mathrm{~mm}$. Detalhes referentes ao experimento podem ser obtidos em Lovato et al. (2004). O segundo experimento, instalado em 1995, está localizado na área experimental da Embrapa Agropecuária do Oeste, sobre um Latossolo Vermelho distroférrico-LVdf (Embrapa, 1999) textura muito argilosa. O clima de ocorrência é o Aw (Köppen), com estação quente e chuvosa no verão e acentuadamente seca no inverno. Descrições mais detalhadas do experimento estão disponíveis em Salton (2005). No quadro 1 são apresentadas características referentes à distribuição granulométrica e mineralogia dos solos.

No experimento do RS, amostrou-se o solo sob preparo convencional (PC) com a sucessão aveia (Avena strigosa)/milho (Zea mays) e, em plantio direto (PD), sob aveia + ervilhaca (Vicia sativa)/milho + caupi (Vigna unguiculata). No experimento do MS, amostrouse o solo em PC na sucessão aveia/soja (Glycine max) e sob pastagem permanente (Pastagem) de braquiária (Brachiaria decumbens). As amostras do PVd foram coletadas em outubro de 2003, no final do ciclo das culturas de inverno. No LVdf, as amostras foram coletadas após a colheita das culturas de verão, em abril de 2004, antecedendo a estação seca.

Com o intuito de obter uma amostra representativa dos tratamentos de manejo de solo, blocos indeformados, da camada de $0-5 \mathrm{~cm}$, foram coletados manualmente nas três repetições de cada experimento. Os agregados de solo foram separados, passados em 
Quadro 1. Distribuição granulométrica e mineralogia dos solos (Inda Junior et al., 2007)

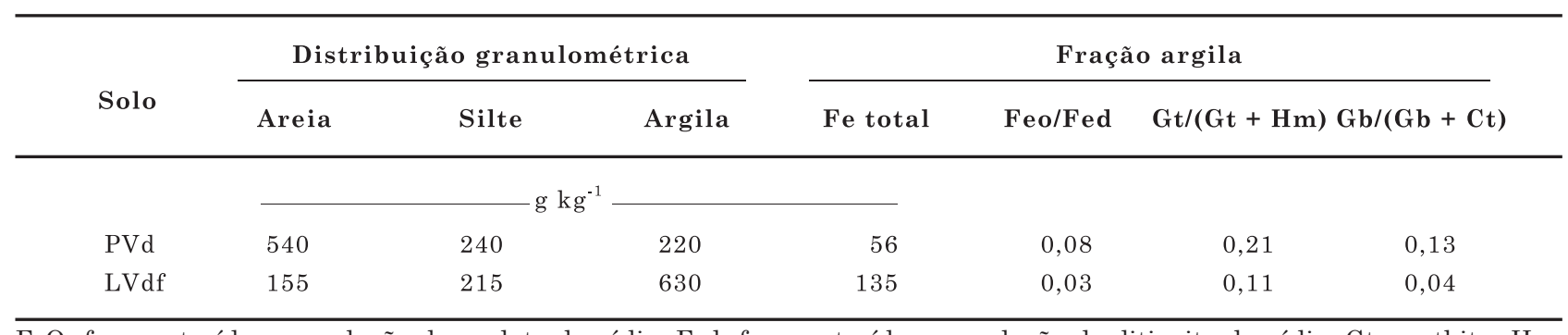

FeO: ferro extraído com solução de oxalato de sódio; Fed: ferro extraído com solução de ditionito de sódio; Gt: goethita; Hm: hematita; Gb: gibbsita; Ct: caulinita; PVd: Argissolo Vermelho distrófico; LVdf: Latossolo Vermelho distroférrico (Embrapa, 1999).

peneira de malha de 9,51 mm (Carpenedo \& Mielniczuk, 1991) e secos ao ar; os materiais obtidos nas três repetições de cada tratamento foram reunidos numa amostra composta de solo. O fracionamento densimétrico da MOS para obtenção da FLL e FLO (Golchin et al., 1994b) foi realizado em triplicata e utilizaram-se soluções de $\mathrm{NaI} 1,8 \mathrm{~g} \mathrm{~cm}^{-3}$ e de PTS $\left[\left(\mathrm{Na}_{6}\left(\mathrm{H}_{2} \mathrm{~W}_{12} \mathrm{O}_{40}\right)\right]\right.$ nas densidades de 1,8, 2,0 e $2,2 \mathrm{~g} \mathrm{~cm}^{-3}$, elaboradas a partir de reagentes das marcas Synth (99,5\%) e Sometu (99,9\%), respectivamente. O fluxograma das etapas do fracionamento é apresentado na figura $1.0 \mathrm{pH}$ da solução de $\mathrm{NaI}$ foi ajustado para 4,0 para igualar-se ao pH da solução de PTS e evitar, portanto, possível solubilização de material orgânico no pH original daquela solução $(>6,0)$. Vinte gramas de solo foram adicionados a $80 \mathrm{~mL}$ de solução de $\mathrm{NaI}$ ou PTS em tubo de centrífuga de $100 \mathrm{~mL}$. O tubo foi fechado com rolha e invertido lenta e manualmente por cinco vezes, visando a liberação da FLL e evitando o rompimento dos agregados. A suspensão foi centrifugada a $2.000 \mathrm{~g}$ por $90 \mathrm{~min}$, e o sobrenadante com a FLL foi filtrado, sob vácuo, em filtro Whatman GF/C previamente quantificado quanto à sua massa. Para retirar o excesso de sal (NaI ou PTS), o filtro + FLL foi lavado com água destilada, sendo seco a $60^{\circ} \mathrm{C}$ por $24 \mathrm{~h}$ e quantificada a massa de filtro + FLL. Para separar a FLO, a solução de NaI ou PTS foi retornada ao tubo que continha o pellet, o qual foi suspenso novamente e submetido à dispersão com ultra-som, mediante energia de $240 \mathrm{~J} \mathrm{~mL}^{-1}$ para o PVd e $410 \mathrm{~J} \mathrm{~mL}^{-1}$ para o LVdf, sendo estes os níveis de energia para obtenção da máxima dispersão desses solos em partículas primárias (Inda Junior et al., 2007). Após a dispersão, a suspensão foi novamente centrifugada (2.000 g por 90 min) e a FLO obtida por filtragem, da mesma forma que a FLL. As soluções de NaI e de PTS foram recuperadas e recicladas segundo Six et al. (1999).

Após obtenção da FLO, o pellet que permaneceu no tubo, o qual corresponde à FP, foi suspenso novamente em água destilada e passado em peneira de $53 \mu \mathrm{m}$, com auxílio de jato de água. O material retido na peneira, composto de areia e MO particulada (MOP), foi transferido para potes de $100 \mathrm{~mL}$ e seco em estufa a $60^{\circ} \mathrm{C}$, sendo essa fração denominada de
MOP residual (Figura 1). As frações densimétricas, a MOP residual e o solo integral foram analisados em relação aos teores de $\mathrm{C}$ pelo método da combustão seca, em analisador Shimadzu-TOC-V CSH. O C da FP foi calculado por diferença entre o C orgânico do solo inteiro e o C da FLL+FLO, sendo, portanto, o C da fração MOP residual considerado como pertencente à FP para fins de discussão do estoque de C dessa fração - isso é o que usualmente ocorre em estudos de fracionamento densimétrico da $\mathrm{MO}$ no solo. Os estoques de $\mathrm{C}$ nas frações físicas e no solo inteiro foram calculados em massa equivalente de solo (Ellert \& Bettany, 1995), considerando o tratamento sob plantio direto (PVd) e pastagem $(\mathrm{LVd})$ como referências.

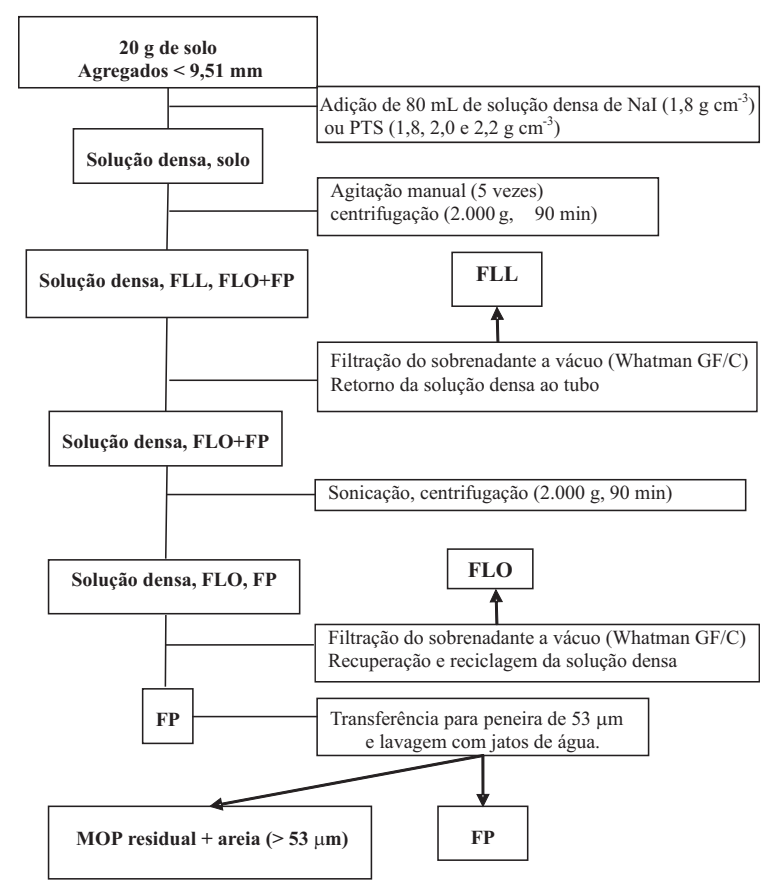

Figura 1. Esquema simplificado do fracionamento físico densimétrico com iodeto de sódio (NaI) e politungstato de sódio (PTS) visando a separação da fração leve-livre (FLL), fração leve-oclusa (FP), fração pesada (FP) e da fração particulada residual (MOP residual) de granulometria tamanho areia. 
Os resultados do fracionamento densimétrico da MO foram submetidos à análise da variância separadamente para cada solo, considerando um delineamento inteiramente casualizado, sendo a diferença entre médias avaliada pelo teste de Tukey a $5 \%$.

\section{RESULTADOS E DISCUSSÃO}

Teores de $\mathrm{C}$ nas frações leve-livre e leve-oclusa e na matéria orgânica particulada residual

Considerando a densidade de $1,8 \mathrm{~g} \mathrm{~cm}^{-3}$ para ambos os produtos, a solução de PTS aumentou de 176 a $727 \%$ o rendimento de $\mathrm{C}$ na FLO, em comparação à solução de NaI, não tendo sido verificado incremento significativo no rendimento de C da FLL (Quadro 2). $\mathrm{Na}$ literatura nacional e internacional não existe estudo sistemático que avalia a eficiência das soluções de NaI e PTS na recuperação da fração leve da MO do solo, apenas uma menção, feita por Shang \& Tiessen (2001), de uma possível menor eficiência do NaI, mas sem que resultados tenham sido apresentados.

A menor eficiência da solução de $\mathrm{NaI}$ em relação à solução de PTS, mencionada por Shang \& Tiessen (2001) e comprovada sistematicamente no presente estudo no que se refere à recuperação da FLO, deve ser resultante da afinidade e formação de complexos de alta estabilidade entre o íon I' e a MO (Yu et al.,
1996; Radlinger \& Heumann, 2000; Schmett et al., 2006). A formação de complexos entre I- e MO resulta provavelmente no aumento de densidade da FLO e redução da eficiência de sua recuperação quando do uso da solução de NaI. Por outro lado, a baixa concentração de grupos funcionais capazes de reagir com o íon I' na estrutura da FLL deve explicar o comportamento similar entre as soluções de NaI e PTS na recuperação dessa fração no solo. Essas hipóteses relativas à causa da menor eficiência da solução de $\mathrm{NaI}$, entretanto, deverão ser testadas em estudos com esse objetivo específico.

Essa subestimação no rendimento de C na FLO quando do uso de solução de $\mathrm{NaI}$ no fracionamento densimétrico da MO do solo resultou em concentração de $\mathrm{C}$ na FLL superior à da FLO em 1,7 vez no PVd e em 2,8 vezes no LVdf, na média dos sistemas de manejo de solo (Quadro 2). No entanto, esse maior estoque de C na FLL, verificado também por diversos outros autores brasileiros (Roscoe et al., 2001; Sohi et al., 2001; Freixo et al., 2002b,c; Pinheiro et al., 2004), não é coerente com os mecanismos de estabilização dessas frações, pois a FLO que apresenta dois mecanismos de estabilização no solo (recalcitrância + oclusão) deveria ter maior teor de C do que a FLL cuja estabilidade advém apenas da recalcitrância intrínseca da biomolécula à degradação microbiana (Sollins et al., 1996). A ausência de efeito do plantio direto e da pastagem, em comparação ao solo em PC,

Quadro 2. Efeito do uso de soluções de iodeto de sódio (NaI) e de politungstato de sódio (PTS) na recuperação de C das frações leve-livre (FLL), leve-oclusa (FLO) e pesada (FP) da matéria orgânica (MO) do solo, e MO particulada residual (MOP residual) em Argissolo Vermelho (PVd) e Latossolo Vermelho (LVdf) submetidos a diferentes sistemas de manejo

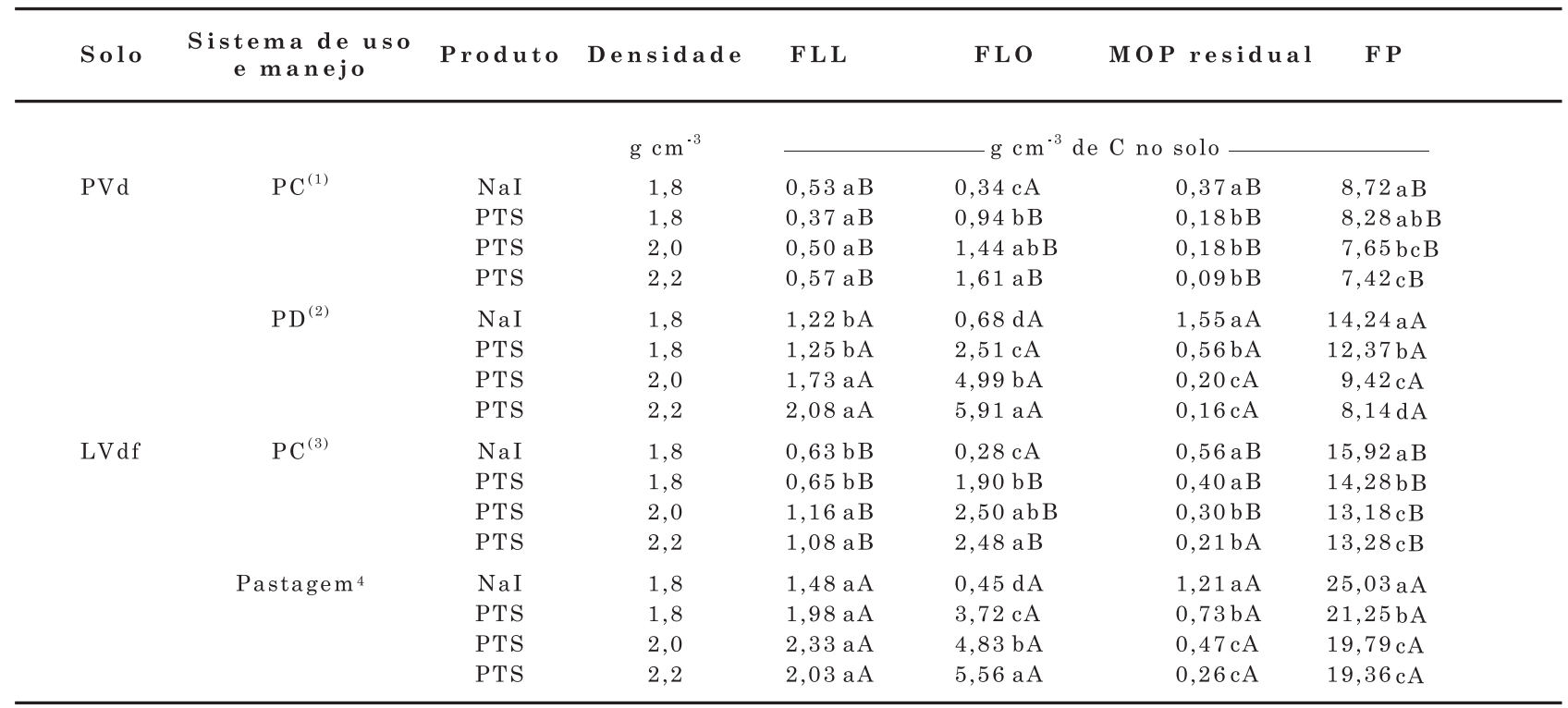

(1) PC: preparo convencional no sistema aveia/milho. ${ }^{(2)} \mathrm{PD}$ : plantio direto no sistema aveia + vica/milho + caupi. ${ }^{(3)} \mathrm{PC}$ : preparo convencional no sistema trigo/soja. ${ }^{(4)}$ Pastagem permanente de Brachiaria decumbens. Médias seguidas pelas mesmas letras minúsculas na coluna comparam densidades e produtos dentro dos sistemas de uso e manejo em cada solo e por letras maiúsculas comparam sistemas de preparo entre si dentro de cada produto e densidade num mesmo solo, não diferindo entre si pelo teste de Tukey a $5 \%$. 
nos teores de C na FLO, no PVd e no LVdf, respectivamente, também não é coerente com a melhoria expressiva usualmente observada na agregação do solo sob esses sistemas de manejo de solo (Silva \& Mielniczuk, 1997a,b; Castro-Filho et al., 1998). Nesse contexto, a conclusão dos resultados obtidos com a solução de NaI, a qual subestima o teor de $\mathrm{C}$ na FLO, leva à interpretação errônea de que a proteção física da MO por oclusão em agregados de solo não é um mecanismo importante de estabilização do C orgânico no solo.

Essas incoerências desapareceram quando da utilização da solução de PTS $1,8 \mathrm{~g} \mathrm{~cm}^{-3}$; a FLO apresentou teor de $\mathrm{C}$ aproximadamente 2,3 vezes maior do que a FLL, na média dos solos e sistemas de manejo (Quadro 2). Além disso, o aumento no teor de C nos sistemas plantio direto e pastagem, calculado em comparação ao solo em PC, foi maior na FLO (1,57 $\mathrm{g} \mathrm{kg}^{-1}$ de C em PD e 1,82 $\mathrm{g} \mathrm{kg}^{-1}$ de C em pastagem) do que na FLL ( $0,88 \mathrm{~g} \mathrm{~kg}^{-1} \mathrm{de} \mathrm{C}^{1} \mathrm{em}$ PD e $1,33 \mathrm{~g} \mathrm{~kg}^{-1}$ de $\mathrm{C}$ em pastagem). Esses resultados evidenciam que a proteção física é um mecanismo relevante de estabilização da $\mathrm{MO}$ em solos brasileiros sob sistemas de manejo sem revolvimento do solo.

O melhor desempenho da solução de PTS $\left(1,8 \mathrm{~g} \mathrm{~cm}^{-3}\right)$ em comparação à solução de $\mathrm{NaI}$ foi verificado não somente pelo aumento da concentração de C da FLO, mas também pela diminuição expressiva no teor de $\mathrm{C}$ na MOP residual (Quadro 2), a qual constitui-se de MOP não recuperada no fracionamento densimétrico como FLL ou FLO. Na média dos sistemas de manejo, o uso da solução de PTS diminuiu o teor de C na MOP residual em $57 \%$ no PVd e em $34 \%$ no LVdf, quando comparado à solução de NaI (Quadro 2). Teores semelhantes ou um pouco superiores de C na MOP residual foram obtidos por Freixo et al. (2002b,c) e Roscoe et al. (2001) em solos brasileiros, utilizando solução de NaI. Entretanto, normalmente esses teores de C na fração areia obtida por peneiramento após o fracionamento densimétrico têm sido considerados baixos, porém, quando considerada a massa da fração, podem ser equivalentes a até mais de $10 \%$ do $\mathrm{C}$ orgânico total (Roscoe et al., 2001), acentuando-se esse efeito em solos arenosos ou franco-arenosos. No presente estudo, o $\mathrm{C}$ separado como MOP residual variou de 3,3 a 9,6 \% do C orgânico total do solo quando se utilizou solução de $\mathrm{NaI}$, ao passo que, quando foi utilizada a solução de PTS $2,0 \mathrm{~g} \mathrm{~cm}^{-3}$, com maior capacidade de recuperação do C na FLO, a quantidade de $\mathrm{C}$ na MOP residual reduziu para apenas 1,6 \% do C orgânico total na média dos solos.

Em adição ao efeito do produto (NaI versus PTS), o aumento de densidade da solução de PTS também resultou em maior rendimento de C em FLL e FLO. O incremento da densidade da solução de PTS de 1,8 para $2,0 \mathrm{~g} \mathrm{~cm}^{-3}$ aumentou o rendimento de C na FLL (38 a $78 \%$ ) e na FLO (30 a $99 \%$ ) nos diferentes solos e sistemas de manejo. Esses resultados seguiram a mesma tendência dos obtidos por Golchin et al. (1994a), que verificaram aumento médio de $26 \%$ na recuperação do $\mathrm{C}$ da FLO pela alteração da densidade da solução de PTS de $1,8 \mathrm{~g} \mathrm{~cm}^{-3}$ para $2,0 \mathrm{~g} \mathrm{~cm}^{-3} \mathrm{em}$ dois solos australianos (12 e $72 \%$ de argila).

De maneira geral, o uso de solução de PTS na densidade de $2,2 \mathrm{~g} \mathrm{~cm}^{-3}$ resultou em recuperação de $\mathrm{C}$ semelhante à obtida com solução de densidade de $2,0 \mathrm{~g} \mathrm{~cm}^{-3}$, indicando que não houve benefício do aumento adicional de densidade da solução de PTS além de $2,0 \mathrm{~g} \mathrm{~cm}^{-3}$. A não-diferença estatística no teor de C na MOP residual entre as soluções de PTS com densidade de 2,0 e $2,2 \mathrm{~g} \mathrm{~cm}^{-3}$ reforça a desvantagem de utilizar solução com densidade superior a $2,0 \mathrm{~g} \mathrm{~cm}^{-3}$ (Quadro 2). Além disso, do ponto de vista prático de obtenção da FLO no fracionamento densimétrico da MO do solo, o pellet obtido com a centrifugação da suspensão utilizando solução de $2,0 \mathrm{~g} \mathrm{~cm}^{-3}$ foi mais firme do que o obtido com a solução de $2,2 \mathrm{~g} \mathrm{~cm}^{-3}$, devido, provavelmente, à maior coesão entre as partículas de solo, o que facilita a obtenção da FLO por meio da filtragem do sobrenadante da suspensão, sem que haja desprendimento do pellet do fundo do tubo de centrífuga.

\section{Magnitude dos mecanismos de estabilização da MO nos solos e efeito dos sistemas de manejo}

A magnitude dos mecanismos de estabilidade da MO no solo foi avaliada pelos estoques de C orgânico nas FLL, FLO e FP obtidas com o uso da solução de PTS 2,0 $\mathrm{g} \mathrm{cm}^{-3}$ (Quadro 3). Nesse sentido, a FLL representou, na média dos sistemas de manejo, $8 \%$ dos estoques de $\mathrm{C}$ orgânico total em ambos os solos, indicando que o mecanismo de recalcitrância molecular da MO no solo tem importância limitada diante da estabilidade decorrente da proteção física da $\mathrm{MO}$ em agregados de solo (23 \% no PVd e $16 \%$ no LVdf) e da sua interação com minerais ( 69 \% no PVd e 76 \% no LVdf).

Entre os sistemas de manejo, verificou-se que a proporção de $\mathrm{C}$ armazenado na FLO da MO do solo foi maior nos solos sob plantio direto e pastagem (31\% no PVd e $18 \%$ no LVdf) do que no solo sob sistema de preparo convencional (11 \% no PVd e $15 \%$ no LVdf), o que se deve à condição sem revolvimento e ao alto aporte de resíduos vegetais, mais favorável à formação e estabilização de agregados de solo. O menor efeito da pastagem na proteção física da MO no LVdf do que do plantio direto no PVd é coerente com a maior resiliência quanto à agregação no $\mathrm{PVd}$ do que no $\mathrm{LVdf}$ (Salton, 2005; Conceição, 2006), o que é decorrente da mineralogia oxídica e maior proporção de hematita e gibbsita no LVdf sob Cerrado do que no solo do RS (Quadro 1).

A proporção de C na FLL também aumentou no solo em PD em comparação ao PC, sendo isso decorrente provavelmente da diminuição na temperatura do PVd em plantio direto e no LVdf sob 
Quadro 3. Estoque de C nas frações leve-livre (FLL), leve-oclusa (FLO) e pesada (FP) da matéria orgânica na camada de 0-5 cm de um Argissolo Vermelho (PVd) manejado sob preparo convencional (PC) e plantio direto (PD) e de um Latossolo Vermelho (LVdf) sob PC e pastagem de Brachiaria (Pastagem) obtidas com solução de politungstato de sódio $2,0 \mathrm{~g} \mathrm{~cm}^{-3}$, e o acúmulo de $\mathrm{C}$ nas respectivas frações em relação ao acúmulo total de $\mathrm{C}$ orgânico total ( $\Delta \mathrm{C}$ fração/ $\Delta \mathrm{COT}$ ) no solo em $\mathrm{PD}$ e pastagem, comparativamente ao solo em $\mathrm{PC}$. Valores entre parênteses correspondem à percentagem de cada fração relativa ao estoque de COT

\begin{tabular}{|c|c|c|c|c|c|c|c|c|}
\hline \multirow{2}{*}{ Solo } & \multirow{2}{*}{ Sistema de manejo } & \multirow{2}{*}{ FLL } & \multirow{2}{*}{ FLO } & \multirow{2}{*}{ FP } & \multirow{2}{*}{ COT } & \multicolumn{3}{|c|}{$\triangle$ fração/ $\triangle \mathrm{COT}$} \\
\hline & & & & & & FLL & FLO & FP \\
\hline & & 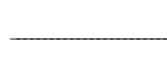 & Mg ha & & - & & & \\
\hline \multirow[t]{2}{*}{ PVd } & $\mathrm{PC}$ & $0,38 \mathrm{~b}(5)$ & $1,08 \mathrm{~b}(15)$ & $5,74 \mathrm{~b}(80)$ & $7,20 \mathrm{~b}$ & & & \\
\hline & $\mathrm{PD}$ & $1,29 \mathrm{a}(11)$ & $3,74 \mathrm{a}(31)$ & 7,07 a $(58)$ & $12,10 \mathrm{a}$ & 0,19 & 0,54 & 0,27 \\
\hline \multirow[t]{2}{*}{ LVdf } & $\mathrm{PC}$ & $0,75 \mathrm{~b}(7)$ & $1,62 b(15)$ & $8,57 \mathrm{~b}(78)$ & $10,94 \mathrm{~b}$ & & & \\
\hline & Pastagem & $1,51 \mathrm{a}(9)$ & $3,14 \mathrm{a}(18)$ & $12,86 \mathrm{a}(73)$ & $17,51 \mathrm{a}$ & 0,12 & 0,23 & 0,65 \\
\hline
\end{tabular}

pastagem (Salton \& Mielniczuk, 1995), a qual diminuiria a taxa de decomposição microbiana dessa fração lábil da MO do solo. Por sua vez, o aumento do C em FLO e FLL resultou na redução da proporção de C na FP da MO do PVd sob plantio direto e LVdf sob pastagem, em comparação ao solo em PC. Essa menor proporção de $\mathrm{C}$ associado aos minerais (FP) decorreu do aumento efetivo da FLO protegida fisicamente, e não significa que a interação da MO com os minerais do solo seja menos importante no solo em PD ou sob pastagem do que no solo em PC, pois, em termos absolutos, ocorreu aumento de $\mathrm{C}$ também na FP em ambos os solos.

O impacto dos sistemas de manejo nos mecanismos de estabilização da MO e o seu reflexo no acúmulo de C orgânico nos solos sob sistemas conservacionistas de manejo foram avaliados pela proporção de aumento dos estoques de $\mathrm{C}$ nas FLL, FLO e FP da MO do solo, em relação ao aumento no estoque total de C orgânico no solo ( $\Delta \mathrm{C}$ fração/ $\Delta \mathrm{C}$ orgânico), cujos resultados são apresentados no quadro 3 . Na média dos dois solos, o acúmulo de $\mathrm{C}$ na FLL foi de aproximadamente $15 \%$ em relação ao acúmulo total de $\mathrm{C}$ orgânico no solo. $\mathrm{O}$ efeito do manejo do solo nessa fração orgânica, que tem na recalcitrância o seu único mecanismo de estabilidade no solo, foi relacionado às alterações no microclima, que tornaram o ambiente menos favorável à decomposição microbiana no solo sob $\mathrm{PD}$ e pastagem do que sob preparo convencional.

O acúmulo de C na FLO representou 54 e $23 \%$ do acúmulo total de $\mathrm{C}$ orgânico verificado nos solos $\mathrm{PVd}$ e LVdf, respectivamente. Esses resultados demonstram que a proteção física em agregados, a qual, juntamente com a recalcitrância molecular, é o principal mecanismo de estabilidade desta fração orgânica no solo, é um importante fator do acúmulo de MO em solos sob plantio direto e pastagem, principalmente naqueles que apresentam resposta maior da formação e estabilidade de agregados ao manejo, como foi o caso do solo menos argiloso e com mineralogia caulinítica do Sul do Brasil.

Possivelmente, as alterações no microclima e a maior proteção física da MO no LVdf aumentaram o tempo de permanência da MO no solo e favoreceram a maior interação do $\mathrm{C}$ orgânico com a fração mineral do solo; 65 \% do acúmulo total de $\mathrm{C}$ orgânico no solo ocorreu na FP, a qual é estabilizada no solo pela sua associação com a fração mineral, além da recalcitrância e proteção física em agregados. No solo PVd - que é menos argiloso, além de caulínitico - a estabilidade química da MO teve menor impacto no acúmulo de $\mathrm{C}$ orgânico do que a proteção física em agregados de solo sob plantio direto.

\section{CONCLUSÕES}

Na densidade de $1,8 \mathrm{~g} \mathrm{~cm}^{-3}$, a solução de iodeto de sódio é menos eficiente na recuperação da fração leveoclusa (intra-agregados) da matéria orgânica do solo do que a solução de politungstato de sódio, as quais não se diferenciam na recuperação da fração leve-livre (interagregados). O aumento da densidade da solução de politungstato de sódio incrementa a capacidade de recuperação das frações leve-livre e leve-oclusa da matéria orgânica. Esse incremento na recuperação das frações é mais expressivo até a densidade de $2,0 \mathrm{~g} \mathrm{~cm}^{-3}$, que é a densidade da solução de politungstato de sódio recomendada em estudos da proteção física da matéria orgânica no solo. A proteção física da matéria orgânica por oclusão em agregados é um importante mecanismo de estabilização e acúmulo de C orgânico em solos tropicais e subtropicais sob plantio direto e pastagem. 


\section{AGRADECIMENTOS}

À Fundação de Amparo à Pesquisa do Estado do Rio Grande do Sul, ao Conselho Nacional de Desenvolvimento Científico e Tecnológico $(\mathrm{CNPq})$ e ao Ministério da Ciência e Tecnologia (MCT), pelo auxílio financeiro no âmbito do projeto PRONEX 04/850.0 "Seqüestro de carbono e mitigação das emissões de gases de efeito estufa por sistemas conservacionistas de manejo e as oportunidades para o agronegócio no RS”. C. Bayer e J. Mielniczuk agradecem ao CNPq a bolsa de produtividade em pesquisa.

\section{LITERATURA CITADA}

BALDOCK, J.A.; OADES, J.M.; WATERS, A.G.; PENG, X.; VASSALLO, A.M. \& WILSON, M. A. Aspects of the chemical-structure of soil organic materials as revealed by solid-state $\mathrm{C}^{13}$ NMR-Spectroscopy. Biogeochemistry, 16:1-42, 1992.

CARPENEDO, V. \& MIELNICZUK, J. Estado da agregação e qualidade de agregados de Latossolos Roxos, submetidos a diferentes sistemas de manejo. R. Bras. Ci. Solo, 14:99$105,1991$.

CASTRO-FILHO, C.; MUZILLI, O. \& PODANOSCHI, A.L. Estabilidade de agregados e sua relação com o teor de carbono orgânico num Latossolo Roxo distrófico, em função de sistemas de plantio, rotações de culturas e métodos de preparo das amostras. R. Bras. Ci. Solo, 22:527538, 1998.

CHENU, C. \& PLANTE, A.F. Clay-sized organo-mineral complexes in a cultivation chronosequence: revisiting the concept of the 'primary organo-mineral complex'. Eur. J. Soil Sci., 57:596-607, 2006.

CHRISTENSEN, B.T. Physical fractionation of soil and organic matter in primary particle size and density separates. Adv. Soil Sci., 20:1-90, 1992.

CHRISTENSEN, B.T. Organic matter in soil - Structure, function and turnover. Copenhagen, 2000. 95p.

CONCEIÇÃO, P.C. Agregação e proteção da matéria orgânica em dois solos do sul do Brasil. Porto Alegre, Universidade Federal do Rio Grande do Sul, 2006. 138p. (Tese de Doutorado)

ELLERT, B.H. \& BETTANY, J.R. Calculation of organic matter and nutrients stored in soils under contrasting management regimes. Can. J. Soil. Sci., 75:529-538, 1995.

EMPRESA BRASILEIRA DE PESQUISA AGROPECUÁRIA . EMBRAPA. Sistema brasileiro de classificação de solos. Rio de Janeiro, Centro Nacional de Pesquisa de Solos, 1999. 412p.

FREIXO, A.A.; CANELLAS, L.P. \& MACHADO, P.L.O.A. Propriedades espectrais da matéria orgânica leve-livre e leve intra-agregados de dois Latossolos sob plantio direto e preparo convencional. R. Bras. Ci. Solo, 26:445-453, 2002a.
FREIXO, A.A.; MACHADO, P.L.O.A.; DOS SANTOS, H.P.; SILVA, C.A. \& FADIGAS, F.S. Estoques de carbono e nitrogênio e distribuição de frações orgânicas de Latossolo do cerrado sob diferentes sistemas de cultivo. R. Bras. Ci. Solo, 26:425-434, 2002b.

FREIXO, A.A.; MACHADO, P.L.O.A.; DOS SANTOS, H.P.; SILVA, C.A. \& FADIGAS, F.S. Soil organic carbon and fractions of a Rhodic Ferralsol under the influence of tillage and crop rotation systems in southern Brazil. Soil Till. Res., 64:221-230, 2002c.

GOLCHIN, A.; OADES, J.M.; SKJEMSTAD, J.O. \& CLARKE, P. Soil-structure and carbon cycling. Aust. J. Soil Res., 32:1043-1068, 1994a.

GOLCHIN, A.; OADES, J.M.; SKJEMSTAD, J.O. \& CLARKE, P. Study of free and occluded particulate organic-matter in soils by solid-state ${ }^{13} \mathrm{C}$ CP/MAS NMR-Spectroscopy and scanning electron-microscopy. Aust. J. Soil Res., 32:285309, 1994b.

GREGORICH, E.G. \& JANZEN, H.H. Storage of soil carbon in the light fraction and macroorganic matter. In: CARTER, M.R. \& STEWART, B.A., eds. Structure and organic matter storage in agricultural soils. Boca Raton, CRC Lewis, 1996. p.167-190.

HASSINK, J.; BOUWMAN, L. A.; ZWART, K. B.; BLOEM, J. \& BRUSSAARD, L. Relationships between soil texture, physical protection of organic matter, soil biota, and C and $\mathrm{N}$ mineralization in grassland soils. Geoderma, 57:105-128, 1993.

INDA JUNIOR, A.V.; BAYER, C.; CONCEIÇÃO, P.C.; BOENI, M.; SALTON, J. \& TONIN, A. T. Variáveis relacionadas à estabilidade de complexos organo-minerais em solos tropicais e subtropicais brasileiros. Ci. Rural, 37:1301-1307, 2007.

LOVATO, T.; MIELNICZUK, J.; BAYER, C. \& VEZZANI, F.M. Adição de carbono e nitrogênio e sua relação com os estoques no solo e com o rendimento do milho em sistemas de manejo. R. Bras. Ci. Solo, 28:175-187, 2004.

PINHEIRO, E.F.M.; PEREIRA, M.G. \& ANJOS, L.H.C. Fracionamento densimétrico da matéria orgânica do solo sob diferentes sistemas de manejo e cobertura vegetal em Paty do Alferes (RJ). R. Bras. Ci. Solo, 28:731-737, 2004 .

RADLINGER, G. \& HEUMANN, K.G. Transformation of iodide in natural and wastewater systems by fixation on humic substances. Environ. Sci. Technol., 34:3932-3936, 2000.

ROSCOE, R. \& BUURMAN, P. Tillage effects on soil organic matter in density fractions of a Cerrado Oxisol. Soil Till. Res., 70:107-119, 2003.

ROSCOE，R.; BUURMAN，P.; VELTHORST， E.J. \& VASCONCELLOS, C.A. Soil organic matter dynamics in density and particle size fractions as revealed by the ${ }^{13} \mathrm{C} /$ ${ }^{12} \mathrm{C}$ isotopic ratio in a Cerrado's oxisol. Geoderma, 104:185202, 2001.

SALTON, J.C. Matéria orgânica e agregação do solo na rotação lavoura-pastagem em ambiente tropical. Porto Alegre, Universidade Federal do Rio Grande do Sul, 2005. 158p. (Tese de Doutorado). 
SALTON, J.C. \& MIELNICZUK, J. Relações entre sistemas de preparo, temperatura e umidade de um Podzólico Vermelho-Escuro de Eldorado do Sul (RS). R. Bras. Ci. Solo, 19:313-319, 1995.

SCHMETT, G.T.; KIMBLE, G.M.; STEINBERG, S.M.; EMERSON, D.W. \& CEREFICE, G. S. 2006. immobilization of fission iodine by reaction with insoluble natural organic matter. Disponível em: <http:// www.nea.fr/html/pt/iempt8/abstracts/Abstracts/ Steinberg_etal.doc> Acesso em: 27 de novembro, 2006.

SHANG, C. \& TIESSEN, H. Sequential versus parallel density fractionation of silt-sized organomineral complexes of tropical soils using metatungstate. Soil Biol. Biochem., 33:259-262, 2001.

SILVA, I.F. Formação, estabilidade e qualidade de agregados do solo afetados pelo uso agrícola. Porto Alegre, Universidade Federal do Rio Grande do Sul, 1993. 126p. (Tese de Doutorado).

SILVA, I.F. \& MIELNICZUK, J. Ação do sistema radicular de plantas na formação e estabilização de agregados do solo. R. Bras. Ci. Solo, 21:113-117, 1997a.
SILVA, I.F. \& MIELNICZUK, J. Avaliação do estado de agregação do solo afetado pelo uso agrícola. R. Bras. Ci. Solo, 21:313-319, 1997b.

SIX, J.; SCHULTZ, P.A.; JASTROW, J.D. \& MERCKX, R. Recycling of sodium polytungstate used in soil organic matter studies. Soil Biol. Biochem., 31:1193-1196, 1999.

SOHI, S.P.; MAHIEU, N.; ARAH, J.R.M.; POWLSON, D.S.; MADARI, B. \& GAUNT, J.L. A procedure for isolating soil organic matter fractions suitable for modeling. Soil Sci. Soc. Am. J., 65:1121-1128, 2001.

SOLLINS, P.; HOMANN, P. \& CALDWELL, B.A. Stabilization and destabilization of soil organic matter: Mechanisms and controls. Geoderma, 74:65-105, 1996.

YU, Z.; WARNER, J.A.; DAHLGREN, R.A. \& CASEY, W.H. Reactivity of iodide in volcanic soils and noncrystalline soil constituents. Geochim. Cosmochim. Acta, 60:49454956, 1996.

ZECH, W.; SENESI, N.; GUGGENBERGER, G.; KAISER, K.; LEHMANN, J.; MIANO, T. M.; MILTNER, A. \& SCHROTH, G. Factors controlling humification and mineralization of soil organic matter in the tropics. Geoderma, 79:117-161, 1997. 